\title{
Low prevalence of handwashing and importance of signage at California county fair animal exhibits
}

Signage showing a link between animal contact and pathogen transmission may lead to increased frequency of handwashing at California county fairs.

by Melissa T. Ibarra, Cheryl L. Meehan, Miles Daniels, Woutrina A. Smith and Martin H. Smith

Online: https://doi.org/10.3733/ca.2021a0015

A nimal exhibitions at county and state fairs bring the public into close proximity with many species of livestock. These interactions create recreational and educational opportunities. However, interfaces between visitors and exhibition animals may also pose public health risks. Livestock may carry zoonotic enteric pathogens, such as Salmonella, Campylobacter, Escherichia coli and Cryptosporidium (Conrad et al. 2017; Hoelzer et al. 2011; Roug et al. 2012). These pathogens can cause serious illnesses in humans, often manifesting as gastrointestinal symptoms, including diarrhea (with or without blood), vomiting, nausea, fever and abdominal cramps (Steinmuller et al. 2006). One study estimated that during a single year in the United States roughly 445,213 illnesses, 4,933 hospitalizations and 76 deaths were caused by the transmission of pathogens (mostly Salmonella, Campylobacter and Cryptosporidium) from animals (Hale et al. 2012).

While exact numbers of illnesses and deaths from enteric diseases originating at county fairs is unknown,

\section{Abstract}

Disease outbreaks among visitors at venues where animals are exhibited, such as animal shows at county fairs or petting zoos, are national public health concerns. Zoonotic disease transmission at fairs can occur through a variety of pathways, including direct contact with livestock and indirect exposure through contact with animals' immediate surroundings. Handwashing can reduce pathogen transmission. The goal of this observational study was to determine rates of handwashing among county fair visitors and to learn whether signage and/or contact with animals were correlated with handwashing practice. The investigation was conducted at four county fairs located across two geographic regions of California. Observations occurred over the course of one summer. Results from our observations of fair visitors revealed a low overall prevalence (5\%) of handwashing behavior. However, fair visitors who made contact with animals were more likely to wash their hands. Additionally, those individuals who walked through barns where handwashing signage was present were significantly more likely to wash their hands than those who visited barns without signage.

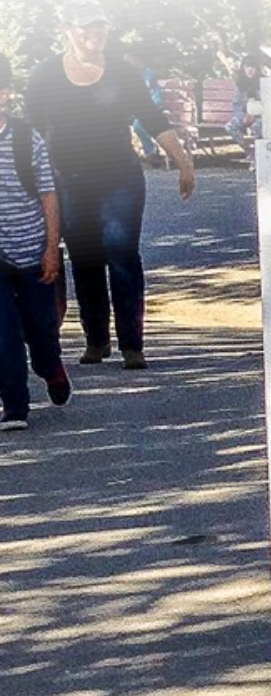

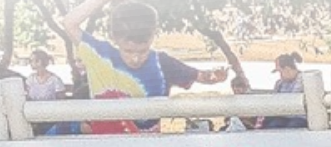

Thank You For Visiting Livestock

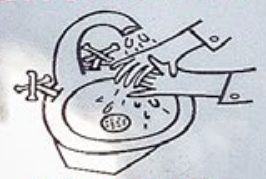

PLEASE REMEMBER TO WASH YOUR HANDS

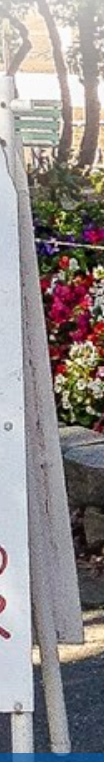

A handwashing station and signage at a California county fair. Results from a recent study of fair visitors indicate a significant positive association between the presence of signage and handwashing practice. Photo: Melissa T. Ibarra. 
multiple reports of outbreaks resulting from disease transmission through animal contact at livestock exhibitions have been reported (Bender and Shulman 2004; CDC 2011; LeJeune and Davis 2004). In California, multiple cases of Shiga toxin-producing E. coli, a contagious bacterial infection with potential severe illness

\section{[M]ultiple reports of outbreaks resulting from disease transmission through animal contact at fairs have been reported....}

consequences, were associated with animal contact at the San Diego County fair in the summer of 2019 (Robbins and Riggins 2019; Sisson 2019). Although any person exposed to zoonotic fecal pathogens is potentially at risk for contracting a disease, some populations are at greater risk for developing a serious or lifethreatening illness, including children (less than five years of age), the elderly (over 65 years of age), pregnant women and individuals with weakened immune systems (LeJeune and Davis 2004).

While disease transmission can occur through a variety of pathways, fecal-oral transmission is the most common route for enteric diseases (CDC 2011). At livestock exhibitions, transmission can occur when visitors make direct physical contact with animals that have pathogen-containing fecal material on their skin/coat, or when visitors come into contact with contaminated

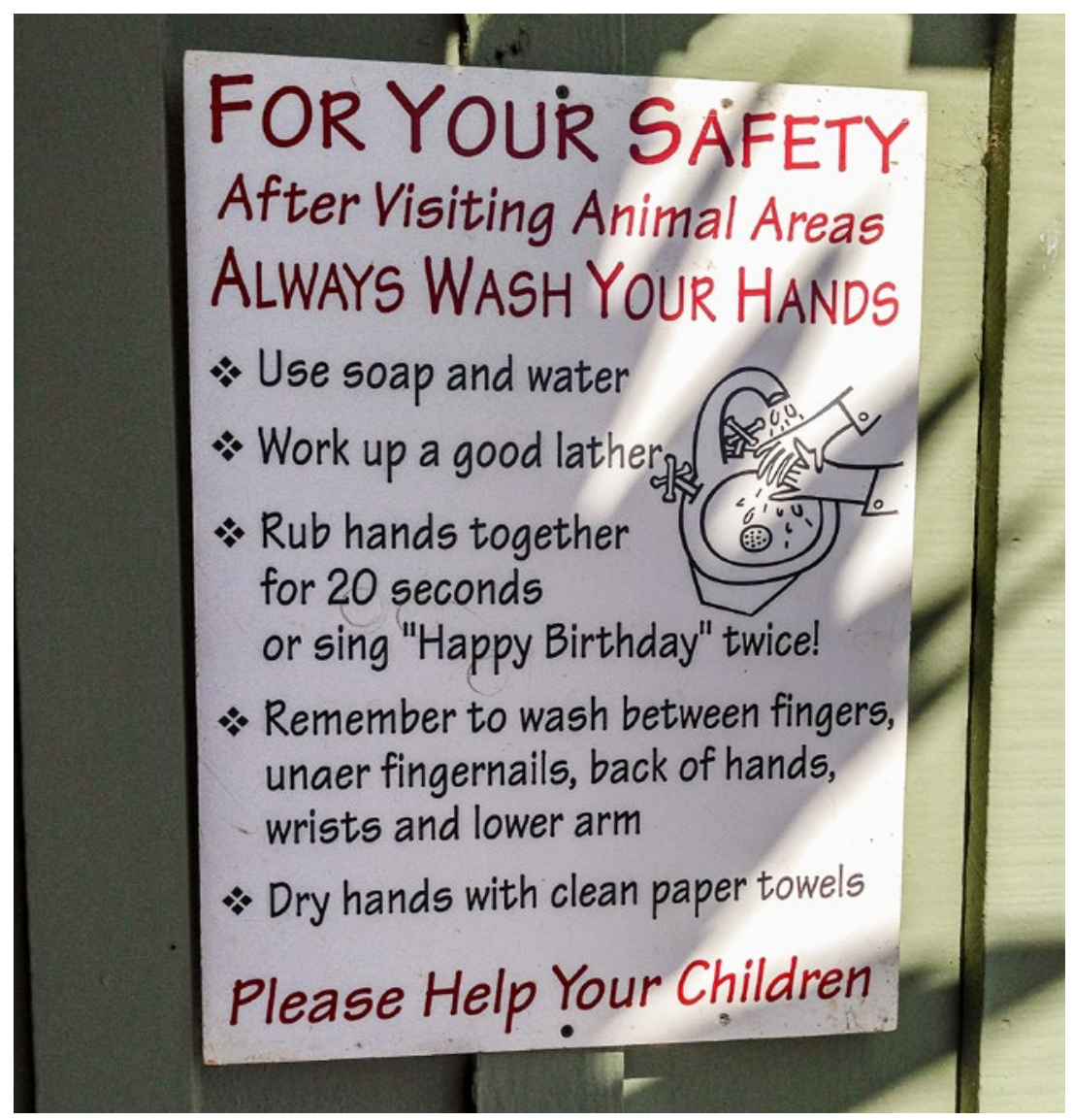

FIG. 1. Example of handwashing signage at the California county fairs included in this study. Photo: Melissa T. Ibarra. feed, bedding, water or pen furnishings. The risk of transmission is increased by several factors associated with exhibitions, including animal transport, increased contact rates among livestock species, frequent livestock handling and the number of animals sharing pen space (Daniels et al. 2021; NASPHV 2013; Thunes and Carpenter 2007).

Some livestock exhibitions offer areas dedicated to animal contact (i.e., petting zoos) while others display animals in barns where visitors are able to make physical contact with livestock and their environments even if not expressly permitted. Exhibit livestock are typically separated from the public by a barrier (e.g., waisthigh walls or railings). However, these barriers do not often prevent fair visitors from interacting with the animals. Visitors' hands can become soiled by pathogencontaining feces through direct contact with animals or by touching contaminated pen furnishings, including bedding, equipment and clothing (Steinmuller et al. 2006). These individuals can inadvertently ingest pathogen-containing feces if they bring their hands to their faces, increasing their risk of developing enteric disease (Erdozain et al. 2013).

Fecal-oral disease transmission is preventable, and appropriate protective measures can be taken to mitigate this risk. Handwashing is a cost-effective option that helps prevent pathogen transmission (CDC 2011; NASPHV 2013), and many fairs provide handwashing stations in livestock areas. Fair visitors, however, do not always make use of handwashing stations, particularly if they are not informed about the role of handwashing in reducing pathogen transmission risks.

While signage has been shown to promote handwashing behavior in health-care settings (Filion et al. 2011), the effectiveness of handwashing signage at California county fairs has not been investigated to date. The goal of this study was to determine if handwashing by California fair visitors after visiting an animal barn was associated with animal contact and/or the presence of handwashing signage.

\section{Study methods}

We conducted this observational study at four county fairs across the state of California during the summer months (July, August, September) of 2015. The fairs were located within two geographic regions of California, the Central Valley (two fairs) and the Central Coast (two fairs). We observed fair visitors specifically for handwashing practice at barns containing livestock. Barn setups varied; some barns contained exhibition animals, others contained animals in petting zoos. However, all observation sites consisted of pens that kept livestock separated from visitors, walkways for visitor traffic and an entrance and exit. Handwashing (sink or alcohol-based hand sanitizer) stations were present at the exits of all barns where observations were made, and handwashing signage was present in some, but not all, barns. 


\section{Handwashing signage}

Signage type varied across barns and fairs (one example is shown in fig. 1). Messaging included both simple content such as "wash your hands" or "wash your hands after petting livestock," as well as more educational descriptions about the importance of handwashing. An example of educational signage included "Help stop the spread of $E$. coli bacterial infection by washing your hands and helping your children wash theirs after any contact with animals." Some signs consisted of a laminated piece of paper created by youth exhibitors; others were permanent and/or prominent fixtures provided by county fair administrators.

\section{Selection of fair visitors}

To select a fair visitor to observe, a researcher in a livestock barn randomly drew a number from 1 to 10 , represented as $x$. This $x$ th adult visitor entering the barn was the designated observee. Once an observational period ended, a new number was drawn, and the process was repeated.

\section{Data collection}

An observational period began when the observed fair visitor reached the barn entrance and ended when the individual exited the barn. We noted whether or not visitors made contact with livestock (i.e., petting or feeding), walked past signage about handwashing, and washed their hands (either with soap and water or with an alcohol-based hand rub) at the barn exit. Data were collected by indicating yes/no for each observed variable. All observations were conducted by a single researcher during regular fair operating hours open to the public. The researcher was dressed in casual attire, was situated outside the direct pathway of visitor foot traffic, maintained distance from visitors during an observational period and carried a clipboard. Research protocols were approved by UC Davis's Institutional Review Board (protocol \#717432-3).

\section{Statistical analysis: Handwashing practices}

We calculated fair visitor prevalence of animal contact (count yes/total), passing signage (count yes/total) and handwashing practice (count yes/total) from raw data. To investigate the relationship between handwashing practice and predictor variables, we ran a logistic regression model with handwashing practice as the response variable. As predictors we used whether or not visitors passed handwashing signage and whether or not they had physical contact with an animal while in the barn. A bivariate model was fit and assessed for multi-collinearity as defined by a variance inflation factor of greater than 10 and a condition index of greater than 30. Regression coefficients were exponentiated such that results are presented as odds ratios (ORs) for ease of interpretation. The OR represents the ratio of the odds of an outcome (handwashing) occurring given a particular exposure (animal contact, signage presence) compared to the odds of the outcome occurring given nonexposure. We conducted our analyses using Jamovi software (version 1.0; The jamovi software project 2019), and we set the significance threshold at an a-level of $P<0.05$.

\section{Associations between signage, animal contact and handwashing}

We observed a total of 337 fair visitors. Of these, 17 (5\%) washed their hands upon exiting a barn; 320 (95\%) did not wash their hands. The rates of handwashing at the four individual fairs were: $7 \%, 2.5 \%, 0 \%$ and $18 \%$ (table 1).

A total of 93 visitors (28\%) were observed to make contact with one or more animals. Of these 93 visitors, 14 (15\%) washed their hands upon exiting the barn. Of the 241 visitors that did not make physical contact with an animal, three (1.2\%) washed their hands upon exiting the barn (table 2).

TABLE 1. Frequency of visitor handwashing by fair

\begin{tabular}{|l|c|c|c|c|}
\hline & \multicolumn{5}{|c|}{ Fair number } \\
\hline Washed hands & $\mathbf{1}$ & $\mathbf{2}$ & $\mathbf{3}$ & $\mathbf{4}$ \\
\hline No & 73 & 79 & 112 & 56 \\
\hline Yes & 5 & 2 & 0 & 10 \\
\hline
\end{tabular}

TABLE 2. Frequency of visitor handwashing by animal contact

\begin{tabular}{|l|c|c|}
\hline \multirow{2}{*}{ Washed hands } & \multicolumn{3}{|c|}{ Animal contact } \\
\hline No & No & Yes \\
\hline Yes & 241 & 79 \\
\hline
\end{tabular}

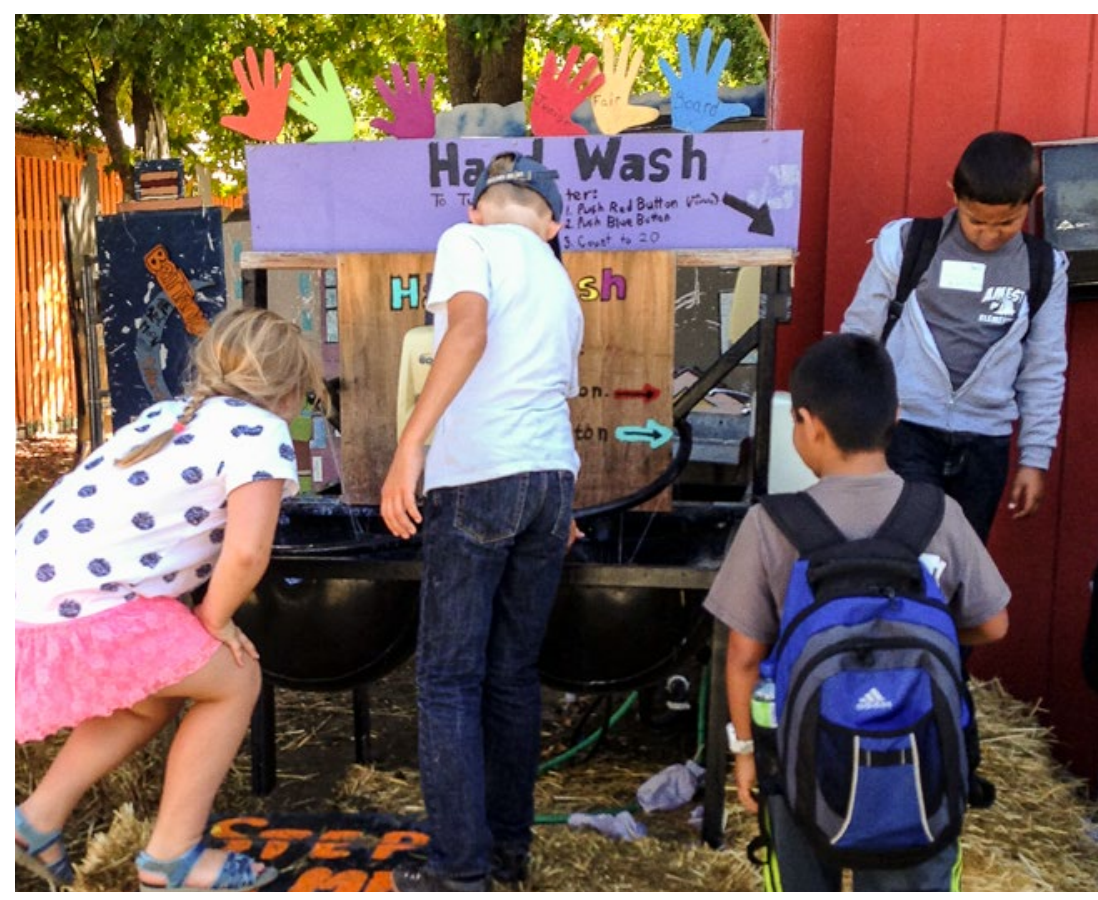

Example of handwashing signage, near a handwashing station. Photo: Melissa T. Ibarra. 
Twenty-nine (9\%) of all visitors walked past handwashing signage. Of these, 10 (34\%) washed their hands upon exit. Of the 308 visitors who did not pass handwashing signage, seven (2\%) washed their hands upon exit (table 3).

Results from the logistic regression analysis (table 4) indicated a significant positive association between the presence of signage and handwashing practice. Results also indicated a significant positive association between animal contact and handwashing practice. The OR for signage was 14.5 , which indicates that visitors who walked past signage were 14 to 15 times more likely to wash their hands than those who did not. The OR for animal contact was 9.5 , which means that people who had contact with animals were nine to 10 times more likely to wash their hands than those who did not.

\section{Understanding the low frequency of handwashing}

Overall, the frequency of handwashing among the fair visitors we observed in this study was very low (5\%). The number of visitors who were exposed to handwashing signage was also low (9\%). Despite these frequencies, our results showed that walking past handwashing signage was associated with a significantly greater likelihood of handwashing, indicating that the presence of signage may be an important strategy in encouraging handwashing practice.

Some of the signage observed in this study incorporated an educational message regarding the health risks associated with animal contact; however, we did not differentiate between signage that was educational in nature and signage that was directional, i.e., "wash your hands," in our analyses. However, this type of analysis would be a beneficial avenue for further study. Although some previous research found no difference in the effectiveness of the type of handwashing signage on handwashing practice (e.g., Erdozain et al. 2013), $\mathrm{Xu}$ et al. (2018) reported recently that awareness of the risk of infectious disease from animals was a reliable predictor of handwashing behavior among adults who made contact with animals in public settings. Signage that promotes such awareness, therefore, might have a positive effect on handwashing behavior.

In interpreting our handwashing results, we could not account for other factors that could affect handwashing behavior, such as visitors' potential knowledge of being observed, prior knowledge of disease transmission risks, fair location, age of visitors or type of handwashing facilities. As such, the efficacy of signage on influencing handwashing behavior should be interpreted cautiously, and further research is warranted. However, based on our findings, our recommendation is that fairs and public exhibitions where animals and people come in close proximity provide an abundance of signage that is visible, offered in multiple languages and provides clear explanations about the risks associated with animal contact and the benefits of handwashing.

Given the general low frequency of handwashing observed in this study, we strongly recommend additional measures be taken to limit health risks to fair visitors. Specifically, limiting visitor access to animal areas and/or having fewer points of entry and exit could direct the flow of visitors past handwashing signage and to handwashing stations. Additionally, fair administrators could consider establishing rules restricting the presence of food or drink inside barns, which could reduce the likelihood of fecal-oral pathogen transmission. Lastly, partnerships among fair administrations, academic institutions, youth agricultural organizations like 4-H and FFA, and local health departments might be advantageous in developing educational announcements, printed materials or video demonstrations - all of which could help reduce disease transmission risks at fairs.

Following completion of this study, our research team partnered with the California Department of Food and Agriculture to produce a series of informational videos highlighting the importance of handwashing as well as other biosecurity best practices for

TABLE 3. Frequency of visitor handwashing by presence of signage

\begin{tabular}{|c|c|c|}
\hline \multirow[b]{2}{*}{ Washed hands } & \multicolumn{2}{|c|}{ Signage } \\
\hline & No & Yes \\
\hline No & 301 & 19 \\
\hline Yes & 7 & 10 \\
\hline
\end{tabular}

TABLE 4. Multivariable assessment of handwashing behavior at the barn exit using logistic regression

\begin{tabular}{|c|c|c|c|c|c|c|c|}
\hline \multirow[t]{2}{*}{ Predictor } & \multirow[t]{2}{*}{ Estimate } & \multirow[t]{2}{*}{ SE } & \multirow[t]{2}{*}{$\mathbf{Z}$} & \multirow[t]{2}{*}{$P$} & \multirow[t]{2}{*}{ Odds ratio } & \multicolumn{2}{|c|}{ 95\% Confidence interval } \\
\hline & & & & & & Lower & Upper \\
\hline Intercept & -4.86 & 0.631 & -7.70 & $<.001$ & 0.00776 & 0.00225 & 0.0267 \\
\hline Signage & 2.68 & 0.583 & 4.59 & $<.001$ & 14.51844 & 4.62961 & 45.5298 \\
\hline Animal contact & 2.25 & 0.679 & 3.31 & $<.001$ & 9.49808 & 2.50805 & 35.9696 \\
\hline
\end{tabular}

Estimates represent the log odds of "Washed hands exit = YES" vs. "Washed hands exit = No." 
visitors and exhibitors during public livestock displays. The videos were created for three discrete audiences: fair administrators (as planning and policy resources), exhibitors (as best practices resources for 4-H and FFA) and fair visitors (as public service announcements).

These videos are available for free download at https:// ucanr.edu/sites/bio-securityeducation/Educational_ Videos/. We recommend the use of these or similar resources whenever possible to help increase knowledge and improve biosecurity practices regarding human/ livestock interactions at fairs and exhibitions.

\section{Opportunities for reducing health risks}

The results from this study highlight the fact that the vast majority of fair visitors are not utilizing a simple and effective tool - handwashing - for reducing the potential for zoonotic disease transmission at county fairs. Therefore, continued efforts to develop and disseminate guidelines for best practices and educational materials for animal exhibitors and the public will be important to assist in the mitigation of disease transmission risks at California county fairs. A combined approach to accomplish this is recommended, including biosecurity education for animal exhibitors (e.g., Smith et al. 2011; Smith et al. 2021; Smith and Meehan, unpublished manuscript), enhanced fair policies designed to limit pathogen transmission, and strategically placed handwashing signage or other forms of fair visitor education like looped videos such as those described previously to increase handwashing practice. Together, these strategies will likely help reduce the public health risks associated with the presence and persistence of fecal-borne pathogens among livestock exhibited at fairs in California (Daniels et al. 2021; Keen et al. 2006; Roug et al. 2012). CA

M.T. Ibarra and M. Daniels were graduate students at UC Davis and now work outside the university; W.A. Smith is Professor and Researcher, Department of Medicine and Epidemiology, UC Davis; C.L. Meehan is Staff Research Associate, Department of Population Health and Reproduction, UC Davis; and M.H. Smith is Specialist in Cooperative Extension, Department of Population Health and Reproduction and Department of Human Ecology, UC Davis.

The authors acknowledge participating California county fairs; the University of California Division of Agriculture and Natural Resources; the University of California School of Veterinary Medicine; Veterinary Medicine Extension; and the Department of Public Health Sciences, UC Davis.

\section{References}

Bender JB and Shulman SA. 2004. Reports of zoonotic disease outbreaks associated with animal exhibits and availability of recommendations for preventing zoonotic disease transmission from animals to people in such settings. J Am Vet Med Assoc 224(7):1105-9. https://doi.org/10.2460/javma.2004.224.1105

[CDC] Centers for Disease Control and Prevention. 2011 Compendium of measures to prevent disease associated with animals in public settings. MMWR Morb Mortal Wkly Rep.60(4):3-22. www.cdc.gov/ $\mathrm{mmwr} / \mathrm{pdf} / \mathrm{rr} / \mathrm{rr} 6004 . \mathrm{pdf}$ (ac cessed on December 1, 2019).

CDC. 2015. Stay healthy at animal exhibits. www.cdc.gov/features/animalexhibits (accessed December 1, 2019)

Conrad CC, Stanford K, NarvaezBravo C, et al. 2017. Farm fairs and petting zoos: A review of animal contact as a source of zoonotic enteric disease. Foodborne Pathog Dis 14(2):59-73. https://doi.org/10.1089/ fpd.2016.2185
Daniels ME, Smith MH, Packham AE, et al. 2021. Associations between faecal pathogen detection, E. coli concentrations and youth exhibitor biosecurity practices at California county fairs. Zoonoses Public Hlth. https://doi.org/10.1111/ zph.12815

Erdozain G, KuKanich K, Chapman B, et al. 2013. Observation of public health risk behaviors, risk communication and hand hygiene at Kansas and Missour petting zoos - 2010-2011. Zoonoses Public HIth 60(4):30410. https://doi.org/10.1111/ j.1863-2378.2012.01531.x

Filion K, KuKanich KS, Chapman $B$, et al. 2011. Observationbased evaluation of hand hygiene practices and the effects of an intervention at a public hospital cafeteria. Am J Infecti Control 39(6):464-70. https://doi.org/10.1016/j. ajic.2010.09.016

Hale CR, Scallan E, Cronquist AB, et al. 2012. Estimate of enteric illness attributable to contact with animals and their environments in the United States. Clin Infect Dis 54(5):S472-9.https:// doi.org/10.1093/cid/cis051
Hoelzer K, Moreno Switt Al, Wiedmann M. 2011. Animal contact as a source of human non-typhoidal salmonellosis. Vet Res 42:34. https://doi. org/10.1186/1297-9716-42-34 Keen JE, Wittum TE, Dunn JR, et al. 2006. Shiga-toxigenic Escherichia coli O157 in agricultural fair livestock, United States. Emerg Infect Dis 12(5):780-6. https://doi.org/10.3201/ eid1205.050984

LeJeune JT, Davis MA. 2004 Reports of zoonotic enteric disease associated with animal exhibits. J Am Vet Med Assoc 224(9):1440-5. https://doi.org/10.2460/javma.2004.224.1440

[NASPHV] National Association of State and Public Health Veterinarians. 2013. Compendium of measures to prevent disease associated with animals in public settings, 2013. J Am Vet Assoc 243(9):1270-80. https://doi. org/10.2460/javma.243.9.1270
Robbins G and Riggins A. 2019. "San Diego County confirms 10th E. coli case in outbreak." The San Diego Union-Tribune. www.sandiegouniontribune.com/news/health/ story/2019-07-10/confirmedcases-of-e-coli-from-san-diegocounty-climbs-to-10 (accessed December 1, 2019).

Roug A, Byrne BA, Conrad PA, Miller WA. 2012. Zoonotic fecal pathogens and antimicrobial resistance in county fair animals. Comp Immunol Microbio Infect Dis 36:306-8. http://doi. org/10.1016/j.cimid.2012.11.006 Sisson P. 2019. "Missed phone calls, changing stories: How E. coli spread at the San Diego County fair." The San Diego Union-Tribune. www.sandiegouniontribune.com/news/ health/story/2019-09-17/san-diego-county-fair-e-coli-outbreak (accessed December 1, 2019). Smith MH, Smith WA, Meehan CL. 2021. 4-H youth advance biosecurity at home and in their communities. Calif Agr 75(1):33-9. https://doi. org/10.3733/ca.2021a0007
Smith $\mathrm{MH}$, Meehan CL, Ma JM, et al. 2011. Bio-security in 4-H Animal Science, a Project Curriculum, Modules 1-3. Division of Agriculture and Natural Resources, University of California. https://anrcatalog.ucanr.edu/ Details.aspx?item $\mathrm{No}=8438$

Steinmuller N, Demma L, Bender JB, et al. 2006. Outbreaks of enteric disease associated with animal contact: Not just a foodborne problem anymore. Clin Infect Dis 43(12):1596-1602. https://doi. org/10.1086/509576

The jamovi project. 2019. jamovi (version 1.6) [computer software]. www.jamovi.org

Thunes $C$ and Carpenter TE. 2007. Biosecurity practices and travel history of individuals exhibiting livestock at the 2005 California State Fair. J Am Vet Assoc 231(4):581-5. https://doi. org/10.2460/javma.231.4.581

Xu W, Cater M, Gravois R, et al. 2018. Animal contact in public settings —risk awareness of enteric pathogens and hand hygiene behaviors. Food Control 91:1-4. https://doi.org/10.1016/j. foodcont.2018.03.030 